\title{
Isolation and Identification of Adenovirus in Hospitalized Children, under Five Years, with Acute Respiratory Disease, in Havana, Cuba
}

\author{
Tania Pumariega, Clara Savón ${ }^{+}$, Mayra Muné, Reynel Cancio, \\ Grehete González, Angel Valdivia, Zoila González, Angel Goyenechea
}

Instituto de Medicina Tropical "Pedro Kourî”, Autopista Novia del Mediodía, km 6, Apartado Postal 601, Marianao 13, Habana, Cuba

Nine Adenovirus (Ad) strains isolated in Cuba, from 128 nasopharingeal swab specimens of children below five years old, with acute respiratory diseases, during 1996 and 1997, were studied by restriction enzyme analysis of genomic DNA with two endonucleases BamH I and Sma I. All different fragment patterns were compared with the respective prototypes. The identified adenoviruses were Ad 1 $(n=4)$, Ad $2(n=1)$ and Ad $6(n=4)$. Males were more frequently infected than females. The analysis of the occurrence of these Adenovirus strains of subgenus $C$ revealed that Ad 1 and Ad 6 were the predominant serotypes in 1996 and in 1997, respectively.

Key words: Adenovirus - acute respiratory infection - restriction analysis - Cuba

To date, 49 Adenovirus serotypes have been recognized. These etiological agents play an important role as pathogens among infants and young children. Adenoviruses of subgenus $\mathrm{C}$, particularly types 1, 2, 5 and 6 are often isolated from cases of respiratory disease mainly in children below five years of age (Li et al. 1996). In Cuba, acute respiratory disease (ARD) constitutes a health major problem, represents the first cause of morbidity and is a leading cause of mortality in the context of all infectious diseases. In view of this situation, a surveillance program has been carried out routinely, showing an increase in the rates of hospitalization due to ARD among children below five years old. It is therefore of major importance to search for what viruses are involved in the etiology of such illness (Goyenechea et al. 1996).

The role of adenoviruses as a cause of upper and lower respiratory tract diseases has not yet been studied thoroughly by enzyme restriction analysis in many countries including Cuba. However, the current classification of isolated strains largely relies on the use of this method (Kajón et al. 1994).

${ }^{+}$Corresponding author. Fax: +53-7-220633/246051. E-mail: clara@ipk.sld.cu

Received 9 September 1999

Accepted 5 May 2000
This report provides the data about the occurrence of adenoviruses infection in children up to five years old hospitalized in the respiratory ward of two pediatric hospitals in Havana city during 1996 and 1997 and the complete identification of isolates by enzyme restriction analysis.

Nasopharingeal swab specimens were obtained from 62 and 66 infants and children below five years old in 1996 and 1997 respectively, who were admitted in the respiratory ward with a clinical diagnosis of ARD. All specimens were taken within the first $24 \mathrm{~h}$ of admission until four days after the onset of symptoms. Specimens were placed in vials with viral transport medium (HanksLactoalbumin solution plus antibiotics) and transported under refrigeration to the laboratory. Viral isolation was performed in HeLa cells obtained from the American type culture collection. Isolated strains were identified by indirect immunofluorescence test using a commercial kit including monoclonal antibodies for RSV, Adenovirus, Influenza $A$ and B, Parainfluenza virus 1, 2 and 3 (Chemicon International Inc, $\mathrm{Ca}$, USA). Specimens were processed according to the manufacturer instructions.

Intracellular viral DNA was extracted using a technique described previously (Li et al. 1991). As based on the previous analysis of subgenus $C$ performed by other authors, pathways were designed for the genomic identification. Thus, two enzymes were used in the digestion of the extracted viral DNA: Sma I and BamH I (Boehringer Mannhein 
Biolab, New England). All enzyme reactions were carried out according to the manufacturer's instructions. Aliquots containing 1 to $2 \mu \mathrm{g}$ of viral DNA were digested with 10 to 15 units of these endonucleases. DNA digests were analyzed in $0.8 \%$ to $1.2 \%$ agarose horizontal gels. In each enzyme reaction Adenovirus standard strains types 1, 2, 5 and 6 were included, as well as a molecular weight marker ( $\lambda$ Hind III and $\phi x$ Hae III Promega).

Of 62 clinical specimen examined from ill infants and children in 1996, 5 (8.1\%) yield Adenovirus typical cytophatogenic effect. Similar findings were noted in $4(6.1 \%)$ of the 66 clinical specimens obtained in 1997. These data agree with those of other authors (Takimoto et al. 1991) who reported a $11.7 \%$ positivity following examination of 94 clinical specimens from children with ARD in Brazil.

Restriction analysis with BamH I and Sma I (Figure) allowed us the complete characterization of three different Adenovirus types. In 1996 the most frequent was Adenovirus type 1, accounting for $80 \%$ of Adenovirus isolated. In a similar analysis carried out in 1997, type 6 was the most prevalent representing $75 \%$ of the isolated strains. An additional interesting finding was that of the nine Adenovirus positive patients, six were male and three were female (Table). These data accord with those of other authors (Kajón et al. 1993). In these studies, in South America, Adenovirus of subgenus $C$ isolated over a seven-year period were found to be much more frequent in male than in female patients. Nevertheless, no significant gender differences were found in regard to the clinical features. This study included all the Adenovirus strains isolated from ARD in $\mathrm{Ha}-$ vana city during two years. Although the sample size was small, this report constitutes the first step towards future studies focusing in the molecular epidemiology of Adenovirus infection in children with ARD in Cuba.
A

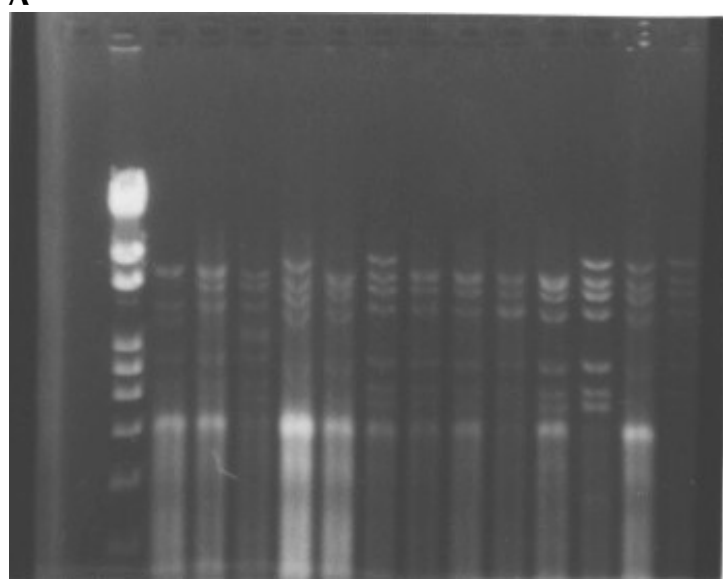

B

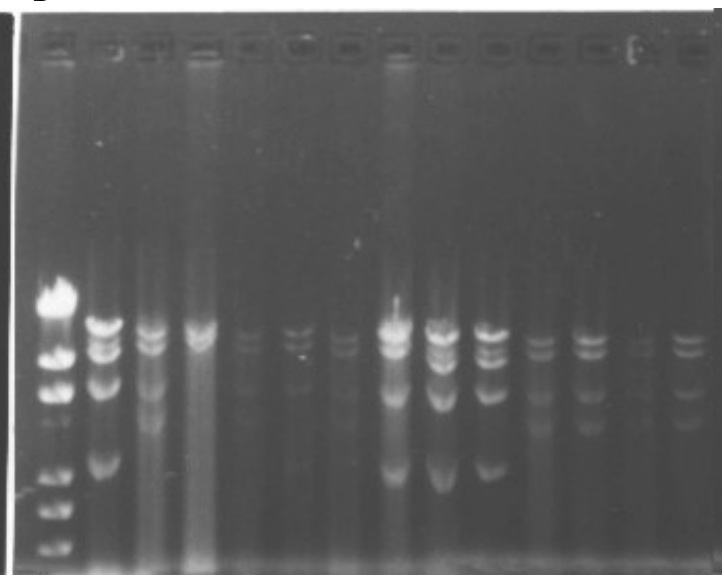

A: restriction pattern with Sma I of the isolated strains (molecular weight pattern, Adv. 1, Adv. 2, Adv. 5, Adv. 6, 100/96, 185/ 96, 245/96, 246/96, 248/96, 83/97, 92/97, 93/97, 167/97); B: restriction pattern with Bam HI of the isolated strains (molecular weight pattern, Adv. 1, Adv. 2, Adv. 5, Adv. 6, 100/96, 185/96, 245/96, 246/96, 248/96, 83/97, 92/97, 93/97, 167/97)

TABLE

Adenovirus strains isolated from children with acute respiratory disease in Havana, Cuba during 1996 and 1997

\begin{tabular}{cllc}
\hline Sample code & Patient's age & Patient's sex & Adenovirus strain \\
\hline $100 / 96$ & 10 months & Male & Ad 1 \\
$185 / 96$ & 8 months & Female & Ad 6 \\
$245 / 96$ & 3 months & Male & Ad 1 \\
$246 / 96$ & 5 years & Male & Ad 1 \\
$248 / 96$ & 1 year & Male & Ad 1 \\
$83 / 97$ & 3 years & Male & Ad 2 \\
$92 / 97$ & 11 months & Female & Ad 6 \\
$93 / 97$ & 11 months & Female & Ad 6 \\
$167 / 97$ & 6 months & & Ad 6 \\
\hline
\end{tabular}




\section{REFERENCES}

Goyenechea A, Razón R, Savón C, Valdivia A, Chacón D, Sarmiento L 1996. Rapid detection of respiratory syncytial virus by indirect immunofluorescence in infants hospitalized with acute respiratory disease. Mem Inst Oswaldo Cruz 91: 489-490.

Kajon A, Wadell G 1994. Genome analysis of South American Adenovirus strains of serotype 7 collected over a 7-year period. J Clin Microbiol 32: 2321-2323.

Kajon A, Suárez MV, Avendaño LF, Hortal M, Wadell G 1993. Genome type analysis of South American adenoviruses of subgenus $C$ collected over a 7-year period. Arch Virol 132: 29-35.
Li QG, Hambraeus J, Wadell G 1991. Genetic relationship between thirteen genome types of Adenovirus 11,34 and 35 with tropisms. Intervirology 32: 338350.

Li Q, Zheng Q, Liu Y, Wadell G 1996. Molecular epidemiology of Adenovirus types 3 and 7 isolated from children with pneumonia in Beijing. J Med Virol 49:170-177.

Takimoto S, Grandien M, Ishida MA, Pereira MS, Paiva TM, Ishimaru T 1991. Comparison of enzyme-linked immunosorbent assay, indirect immunofluorescence assay, and virus isolated for detection of respiratory viruses in nasopharingeal secretions. J Clin Microbiol 29: 470-474. 